\title{
Diagnóstico bacteriológico de tuberculosis pulmonar mediante fibrobroncoscopía en pacientes con VIH
}

\author{
CARLOS PEÑA M.*, MAURICIO CÉSPED G.*, MARCELO WOLFF R.**, \\ FRANCISCA ÁLVAREZ V.***, CAROLINA GARAY B.***, MARILUZ MEDINA P.***, \\ JENNIFER ZÚNIIGA S.**** y ADOLFO CÉSPED Z.*****
}

\section{Bacteriological diagnosis of lung tuberculosis through bronchoscopy in HIV-infected patients}

Tuberculosis can be lethal in HIV infected people. Lung is the organ most frequently involved, but clinical and radiological features are not typical of the disease. Diagnostic certification demands acid-fast bacillus microscopy and mycobacterial cultures on sputum. Some patients need bronchoscopy to obtain samples due to insufficient sputum. We reported a 9.1\% diagnostic yield using bronchoscopy. Clinical suspicion before bronchoscopy had low positive predictive value of tuberculosis (10.8\%). $47.8 \%$ of tuberculosis cases were not suspected before this procedure. Tuberculosis patients showed $C D 4 \leq 200$ cells $/ m L$ (48.8 in average) and less use of ART (antiretroviral therapy). Cultures contributed to the diagnosis of $35 \%$ of tuberculosis cases but with a delay of 30 days. Induced sputum is a less costly alternative to bronchoscopy with a similar diagnostic yield.

Key words: Tuberculosis, HIV, bronchoscopy.

\section{Resumen}

La tuberculosis puede ser letal en pacientes infectados por VIH. El compromiso pulmonar es más frecuente en ellos y su cuadro clínico-radiológico no es típico de la enfermedad. El diagnóstico se confirma con baciloscopía y cultivo de Koch en esputo. Los pacientes sin esputo pueden requerir broncoscopía. Encontramos un rendimiento de 9,1\% en diagnóstico de tuberculosis pulmonar por broncoscopía. La sospecha clínica de tuberculosis previa a broncoscopía tuvo bajo valor predictivo positivo (10,8\%). 47,8\% de los pacientes con Tuberculosis no fueron sospechados antes de la broncoscopía por lo que recomendamos este procedimiento en pacientes VIH con alteraciones radiológicas y sintomas respiratorios. Los casos de tuberculosis tenian $C D 4 \leq 200$ células $/ m L$ (promedio 48,8) y menos uso de TAR: terapia antiretroviral. El 35\% de los casos de tuberculosis se diagnosticó por cultivo (demora mínima de 30 días). El esputo inducido es una alternativa a la broncoscopía menos costosa $y$ de similar rendimiento.

Palabras clave: Tuberculosis, VIH, broncoscopía.

\section{Introducción}

La tuberculosis (TBC) es una infección ampliamente distribuida en el mundo. En 1993 la Organización Mundial de la Salud informó que esta enfermedad constituía una emergencia sanitaria mundial. En 2011 se reportaron cerca de 9 millones de nuevos casos de TBC en el mundo y se tuvo que lamentar la muerte de 1,4 millones de personas por esta causa ${ }^{1}$. La infección por virus de inmunodeficiencia humana adquirida (VIH) constituye un factor de riesgo de desarrollar TBC, estimándose en 20 veces más la probabilidad de reactivación de una $\mathrm{TBC}$ latente en personas

\footnotetext{
* Médicos broncopulmonares, Hospital Clínico San Borja Arriarán.

** Médico infectólogo, Hospital Clínico San Borja Arriarán.

*** Alumnos de medicina, Facultad de Medicina Centro, Universidad de Chile.

**** Tecnóloga de microbiología, Hospital Clínico San Borja Arriarán.

***** Alumno de medicina, Universidad del Desarrollo.
} 
con infección por $\mathrm{VIH}^{2}$. Se ha señalado además que la TBC es la principal causa de muerte por enfermedad infecciosa en estas personas ${ }^{3}$. De los fallecidos por TBC entre 26 y $30 \%$ corresponden a personas seropositivas ${ }^{4}$. En autopsias de pacientes con VIH, la TBC estaba presente en 30 a $79 \%$ de ellos 5 .

La TBC en infectados por VIH produce un deterioro inmunitario al comprometer los linfocitos CD4 y ocasiona una progresión del curso de la infección por $\mathrm{VIH}^{6}$. A nivel mundial la coinfección de TBC con VIH es de $13 \%{ }^{7}$. En Chile fue de $5,8 \%$ en el año $2012^{8}$. La TBC pulmonar es la infección más importante desde el punto de vista de salud pública ya que trasmite la enfermedad en la comunidad a través de la eliminación de bacilos mediante la tos. La presencia de TBC pulmonar en personas con infección por VIH determina un estadío clínico de inmunosupresión (estadío 3) ${ }^{9}$. Las presentaciones clínicas y radiológicas de la TBC pulmonar no son las habituales de esta afección.

El diagnóstico de confirmación de la TBC pulmonar se realiza con la detección del bacilo de Koch mediante la baciloscopía (BK) y el cultivo de Koch (CK) en muestras de expectoración. Sin embargo, la sensibilidad de la baciloscopía es menor del $50 \%$. Es necesario un recuento superior a 5.000 bacilos por $\mathrm{mL}$ de muestra de esputo para que la $\mathrm{BK}$ resulte positiva ${ }^{10}$. El CK tiene mayor sensibilidad ya que es capaz de detectar la presencia de hasta 10 a 100 bacilos por $\mathrm{mL}$ de muestra; sin embargo, es un proceso de diagnóstico tardío por el lento crecimiento del bacilo ( 30 a 60 días) ${ }^{11}$. Algunos pacientes con alteraciones radiológicas y síntomas respiratorios infecciosos no logran producir muestras satisfactorias de expectoración para efectuar $\mathrm{BK}$ y $\mathrm{CK}$ y se recurre a la fibrobroncoscopía (FBC) y al esputo inducido (EI) como una manera de obtener muestras respiratorias adecuadas, con rendimientos variables entre 12 y $38 \%$ para la FBC y 19 y $34 \%$ para el EI. La sensibilidad del diagnóstico por cultivo de micobacterias es de $73 \%$ para la FBC y $87 \%$ para el EI y el valor predictivo negativo del cultivo obtenido por FBC es de $91 \%$ contra $96 \%$ del $\mathrm{EI}^{12,13}$.

El Servicio de Salud Metropolitano Central (SSMC) tiene una alta incidencia de TBC (16,9 por 100.000 habitantes $)^{14}$, superior al promedio nacional $(12,8 \text { por } 100.000 \text { habitantes })^{15}$ y una proporción de coinfección con VIH de 1\% el año 2011. En este servicio se han diagnosticado casos de TBC pulmonar en infectados con VIH utilizando la FBC, por lo que es necesario conocer el rendimiento de esta técnica e identificar los factores asociados a la probabilidad de diagnóstico de
TBC. Se elaboró con este propósito un protocolo de investigación destacando los aspectos de confidencialidad de los pacientes con infección por VIH. Este protocolo fue autorizado por el Comité de Ética del Servicio de Salud Metropolitano Central.

\section{Pacientes y Método}

Se revisaron los registros de las $\mathrm{FBC}$ realizadas entre el 7 de diciembre del año 2000 y el 27 de julio del año 2011 en la unidad de respiratorio del Hospital San Borja Arriarán y se seleccionaron los procedimientos con el diagnóstico asociado de infección por VIH. Se revisaron las fichas clínicas de estos pacientes y se consignaron los antecedentes de edad, género, indicación de la FBC, tiempo de evolución de la infección por VIH, antecedentes de uso de terapia antiretroviral (TAR), síntomas clínicos, presencia de candidiasis orofaríngea, recuento de linfocitos CD4 y descripción de las imágenes radiológicas torácicas. Se revisaron en el libro de registros de baciloscopías del laboratorio de microbiología del hospital San Borja Arriarán los resultados de la baciloscopía y cultivo de Koch de las muestras obtenidas por la FBC. Se construyó una base de datos sustituyendo la identidad de los pacientes por un número correlativo para proteger su identidad y mantener la confidencialidad.

\section{Criterios de exclusión}

Se excluyeron del estudio los pacientes que en el momento de la realización de la $\mathrm{FBC}$ se encontraban en tratamiento por TBC (información obtenida de los registros del Programa de Control de la Tuberculosis del SSMC), aquellos en los cuales la micobacteria identificada por la BK correspondía a un bacilo ambiental no tuberculoso (atípico) y los pacientes en los cuales no se documentó la infección por VIH.

\section{Procedimiento de FBC}

Las FBC fueron realizadas por especialistas broncopulmonares del hospital utilizando un broncoscopio Olympus. La técnica se realizó con anestesia tópica (dimecaína al 4\%) y se recogieron muestras bacteriológicas para estudio de micobacterias, bacterias, hongos y Pneumocystis jiroveci en todos los pacientes. Las muestras se enviaron al laboratorio antes de los $30 \mathrm{~min}$ posteriores a su recolección.

\section{Procesamiento de muestras respiratorias}

Las muestras de secreción broncoaspirada fueron procesadas en el laboratorio de microbiología 
del hospital clínico San Borja Arriarán por tecnólogos acreditados por el Instituto de Salud Pública de Chile. Se utilizó la tinción de Ziehl-Neelsen tradicional. Para la observación microscópica se utilizó un microscopio binocular (Zeiss), con oculares de 20x y objetivo de inmersión (100x). Se utilizó el criterio de informe recomendado por la Organización Panamericana Sanitaria observando la presencia de bacilos ácido alcohol resistentes (BAAR) en el extendido y promediando el recuento de BAAR de los campos de observación. Para el cultivo del bacilo se utilizó un medio de cultivo sólido de Lowenstein-Jensen (Beckton Dickinson). Las muestras se depositaron en una cámara para lectura a 30 y 60 días informando el número de colonias en intervalos de 1 a 50 colonias, 50 a 100 colonias y más de 100 colonias (incontables colonias).

La información recolectada se incorporó a una planilla Excel y se compararon los antecedentes de uso de TAR, tiempo de evolución conocido de la infección por VIH, síntomas clínicos, recuentos de CD4, presencia de candidiasis orofaríngea y hallazgos radiológicos de los pacientes con y sin TBC pulmonar. Se utilizó para evaluar la información cálculos de distribución de frecuencias, promedios, rangos de variación, validez de pruebas diagnósticas (sensibilidad y especificidad) y como pruebas de significación estadística el test de $\chi^{2}$.

\section{Resultados}

Se registraron $255 \mathrm{FBC}$ realizadas en el período contemplado en el estudio, ninguno de estos casos figuraba como TBC pulmonar en los registros oficiales del Programa de Control de la TBC del Servicio de Salud. Se excluyeron dos casos con micobacterias ambientales y un caso en que se descartó la infección por VIH. De los 252 casos incluidos en el estudio, 216 eran hombres $(85,7 \%)$ y 36 mujeres, con un promedio de edad de 38,4 años y un rango que fluctuó entre los 20 y 67 años. En el 54,36\% se conoció el nivel de
CD4 (137 casos), siendo su promedio de 156,4 células $/ \mathrm{mL}$. El $67,9 \%$ tenía un recuento de CD4 $\leq 200$. Se encontró candidiasis en $34,5 \%$ (87 de 146 casos con pesquisa dirigida). En $88,4 \%$ se registró un nivel importante de inmunosupresión (172 casos) caracterizado por candidiasis orofaríngea y/o recuentos de CD4 $\leq 200$ células $/ \mathrm{mL}$. En 144 pacientes existía registro de uso de TAR $(47,2 \%)$.

Se conoció el tiempo de evolución de la infección por VIH en 151 casos, que fue de 36 meses en promedio (Tabla 1). En 26 casos existía la presencia de sarcoma de Kaposi $(10,3 \%)$ y en 74 casos la carga viral era $\geq 50.000$ copias de RNA.

El síntoma más frecuente fue la tos $(47,6 \%)$ y la fiebre $(41,7 \%)$. La presencia de expectoración sólo alcanzó a 37,3\% (Tabla 1). En 58,7\% (148 casos) se obtuvo información de la radiografía de tórax. El $88,5 \%$ de las radiografías torácicas mostraba alteraciones radiológicas; las más frecuentes correspondieron a opacidades localizadas o difusas $(81,7 \%)$ pero $11,5 \%$ tenían radiografía normal.

\section{Casos $\mathrm{VIH}+$ con tuberculosis pulmonar bacteriológicamente demostrada}

En el registro del laboratorio se identificaron 23 casos de TBC entre las 252 FBC (Tabla 2), lo que da un rendimiento diagnóstico de $9,1 \%$ (Figura 1). En 15 casos la BK fue positiva y en 8 casos sólo el $\mathrm{CK}$ fue positivo. Los pacientes con TBC correspondían a 20 hombres $(87 \%)$ y 3 mujeres, con un promedio de edad de 41,9 años y un rango que fluctuó entre los 26 y 60 años. E1 $43,5 \%$ de los casos de TBC eran pacientes hospitalizados, con un rendimiento diagnóstico de la FBC de 8,8\% (10 de 114) en ellos. El 56,5\% de los casos de TBC eran ambulatorios con un rendimiento diagnóstico de la $\mathrm{FBC}$ de 9,45\% (13 de 138). En 13 casos $(56,5 \%)$ se conoció el nivel de CD4, siendo su promedio de 48,76 células $/ \mathrm{mL}$. Todos tenían un recuento de CD4 $\leq 200$ células/ $\mathrm{mL}$. El $34,78 \%$ tenía candidiasis; sólo $21,7 \%$ recibía TAR.

Tabla 1. Tiempo conocido de evolución de la infección por VIH

\begin{tabular}{|lccccc|}
\hline & $\begin{array}{c}\text { Total con registro } \\
\text { de datos }\end{array}$ & $\begin{array}{c}\text { Sin TBC } \\
\text { pulmonar }\end{array}$ & \multicolumn{3}{c|}{ Con TBC pulmonar } \\
BK+ & 151 & 136 & 11 & 4 & 15 \\
Número de casos & 36 & 36 & 30 & 5 & 23 \\
Promedio de tiempo con VIH (meses) & $0-180$ & $0-216$ & $0-192$ & $0-10$ & $0-192$ \\
\hline Rango de tiempo con VIH (meses) & & & Total \\
\hline
\end{tabular}

BK: baciloscopía; CK: cultivo bacilo de Koch. 


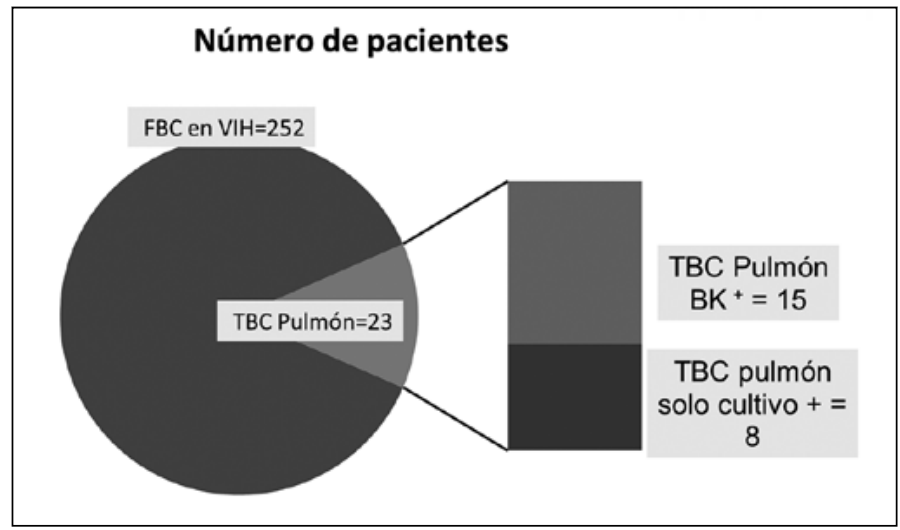

Figura 1. Rendimiento de diagnóstico bacteriológico de tuberculosis. FBC: fibrobroncoscopía. TBC: Tuberculosis. BK: Baciloscopía.

Tabla 2. Características de los casos de tuberculosis

\begin{tabular}{|c|c|c|c|c|c|c|c|c|c|c|c|c|}
\hline Casos & Género & Edad & BK & $\begin{array}{c}\text { Sólo cul- } \\
\text { tivo + }\end{array}$ & $\begin{array}{l}\text { Tm- } \\
\text { VIH }\end{array}$ & $\begin{array}{c}\text { Etapa } \\
\text { VIH }\end{array}$ & CD4 & $\begin{array}{c}\text { Carga } \\
\text { viral }\end{array}$ & TAR & $\begin{array}{l}\text { Tm- } \\
\text { TAR }\end{array}$ & $\begin{array}{l}\text { Candi- } \\
\text { diasis }\end{array}$ & $\begin{array}{c}\text { S. } \\
\text { Kaposi }\end{array}$ \\
\hline 1 & M & 40 & - & $+30 \mathrm{~d}$ & 10 & $\mathrm{C} 3$ & NR & 120.000 & - & - & + & + \\
\hline 2 & M & 48 & + & & 1 & $\mathrm{C} 3$ & 74 & 720.000 & - & - & - & - \\
\hline 3 & M & 40 & + & & 3 & $\mathrm{C} 3$ & 72 & 100.000 & - & - & + & - \\
\hline 4 & M & 54 & - & $+30 \mathrm{~d}$ & 0 & $\mathrm{C} 3$ & 41 & 8.300 & - & - & - & - \\
\hline 5 & $\mathrm{M}$ & 59 & + & & 1 & $\mathrm{C} 1$ & 28 & 2.300 .000 & - & - & + & - \\
\hline 6 & M & 56 & + & & 2 & $\mathrm{C} 3$ & 40 & NR & - & - & + & - \\
\hline 7 & M & 32 & - & $+30 \mathrm{~d}$ & 60 & $\mathrm{C} 3$ & 40 & 15.000 & + & 33 & + & - \\
\hline 8 & M & 38 & + & & 25 & $\mathrm{C} 3$ & 10 & LDE & + & 12 & - & + \\
\hline 9 & $\mathrm{~F}$ & 48 & - & $+60 \mathrm{~d}$ & 0 & NR & NR & NR & - & - & + & - \\
\hline 10 & M & 47 & - & $+30 \mathrm{~d}$ & 0 & $\mathrm{C} 3$ & 62 & 6.400 .000 & - & - & - & - \\
\hline 11 & $\mathrm{M}$ & 31 & + & & NR & NR & NR & NR & NR & NR & NR & NR \\
\hline 12 & $\mathrm{~F}$ & 44 & + & & 1 & $\mathrm{C} 3$ & NR & NR & - & - & - & - \\
\hline 13 & $\mathrm{~F}$ & 33 & + & & NR & NR & NR & NR & NR & NR & NR & NR \\
\hline 14 & $\mathrm{M}$ & 26 & - & $+30 \mathrm{~d}$ & 8 & $\mathrm{C} 3$ & 0 & 88.000 & - & - & - & - \\
\hline 15 & $\mathrm{M}$ & 47 & - & $+60 \mathrm{~d}$ & NR & NR & NR & NR & NR & NR & NR & NR \\
\hline 16 & M & 33 & - & $+60 \mathrm{~d}$ & 112 & $\mathrm{C} 3$ & 269 & 210.000 & + & 36 & - & + \\
\hline 17 & M & 60 & + & & 12 & $\mathrm{C} 2$ & 31 & 180.000 & - & - & + & - \\
\hline 18 & $\mathrm{M}$ & 45 & + & & 21 & $\mathrm{C} 3$ & 30 & 390.000 & + & 21 & + & + \\
\hline 19 & M & 27 & + & & 0 & $\mathrm{C} 3$ & 114 & 650.000 & - & - & - & - \\
\hline 20 & $\mathrm{M}$ & 37 & + & & 4 & $\mathrm{C} 3$ & 121 & 370.000 & - & - & - & - \\
\hline 21 & $\mathrm{M}$ & 28 & + & & 72 & $\mathrm{C} 3$ & 47 & 70.000 & + & 72 & - & - \\
\hline 22 & M & 45 & + & & 3 & $\mathrm{C} 3$ & 11 & 380.000 & - & - & - & + \\
\hline 23 & M & 46 & + & & 192 & $\mathrm{C} 3$ & 115 & 41.000 & + & 192 & - & - \\
\hline
\end{tabular}

Tm-VIH: meses con VIH previo a fibrobroncoscopía (FBC). Tm-TAR: meses de TAR (Tratamiento antirretrovial) previo a FBC. M: masculino; F: Femenino; BK: baciloscopía. 
Tabla 3. Perfil clínico de pacientes con infección por VIH

\begin{tabular}{|c|c|c|c|c|c|c|}
\hline & \multicolumn{2}{|c|}{$\begin{array}{l}\text { Total pacientes } \\
\mathbf{n}=\mathbf{2 5 2}\end{array}$} & \multicolumn{2}{|c|}{$\begin{array}{c}\text { Sin TBC pulmonar } \\
\mathbf{n}=229\end{array}$} & \multicolumn{2}{|c|}{$\begin{array}{c}\text { Con TBC pulmona } \\
n=23\end{array}$} \\
\hline & $\mathbf{n}$ & $\%$ & $\mathbf{n}$ & $\%$ & $\mathbf{n}$ & $\%$ \\
\hline Fiebre $>37,5^{\circ} \mathrm{C}$ axilar & 105 & 41,7 & 91 & 39,7 & 14 & 60,9 \\
\hline Tos & 120 & 47,6 & 104 & 45,4 & 16 & 69,6 \\
\hline Expectoración & 94 & 37,3 & 82 & 35,8 & 12 & 52,2 \\
\hline Baja de peso $>10 \%$ & 78 & 30,9 & 60 & 26,2 & 14 & 60,9 \\
\hline Sudoración vespertina & 65 & 25,8 & 55 & 24 & 10 & 43,5 \\
\hline Adenopatías & 36 & 14,3 & 30 & 13,1 & 6 & 26,1 \\
\hline
\end{tabular}

Tabla 4. Comparación estado inmunitario pacientes VIH+ con y sin tuberculosis pulmonar

\begin{tabular}{|lcc|}
\hline & $\begin{array}{c}\text { Con TBC } \\
\text { pulmonar }\end{array}$ & $\begin{array}{c}\text { Sin TBC } \\
\text { pulmonar }\end{array}$ \\
\hline Promedio CD4 (céls/mL) & 48,76 & 169 \\
\% con CD4 $\leq 200$ (céls/mL) & 100 & 64,52 \\
\% candidiasis orofaríngea & 34,78 & 34,50 \\
\% con TAR* & 21,7 & 49,6 \\
\hline
\end{tabular}

*TAR: Tratamiento antirretroviral; céls: células.

El tiempo promedio de evolución conocida de la infección por VIH en 15 pacientes con TBC pulmonar fue de 23 meses. Los pacientes con TBC pulmonar demostrada por cultivo tenían el menor tiempo de evolución de la infección por VIH (5 meses) y 17,4\% tenía sarcoma de Kaposi. En los casos con carga viral conocida (10 casos) el promedio de CD4 fue $\geq 50.000$. células/ $\mathrm{mL}$. Entre los casos con TBC, los síntomas más frecuentes fueron la tos $(69,6 \%)$, fiebre $(60,9 \%)$ y baja de peso $(60,9 \%)$. La presencia de expectoración sólo alcanzó al 52,2\% (Tabla 3). 43,4\% de ellos tenía un tiempo de evolución de la tos de menos de 6 meses y $21,7 \%$ de menos de 1 mes. En 16 casos se dispuso de la imagen radiológica torácica $(69,6 \%)$ cuyas alteraciones radiológicas más frecuentes fueron las opacidades pulmonares $(81,3 \%)$ y los nódulos pulmonares $(12,5 \%)$. Un paciente tenía radiografía de tórax normal $(6,3 \%)$.

\section{Casos VIH+ sin tuberculosis pulmonar bacteriológicamente demostrada}

Doscientos veintinueve pacientes no presentaron TBC pulmonar, 196 eran hombres $(85,6 \%)$ y 33 mujeres, con un promedio de edad de 38 años y rango de 20 a 67 años. En 54,1\% se conoció el nivel de CD4 y su promedio fue de 169 células/ $\mathrm{mL} ; 64,4 \%$ tenía recuento $\leq 200$ células $/ \mathrm{mL}$. Se encontró candidiasis en 34,5\%. En 97 casos se pudo determinar que el $42,4 \%$ tenía un nivel de compromiso inmunitario importante.

El tiempo promedio de evolución de la infección por VIH en 136 casos fue de 36 meses. Los síntomas más frecuentes fueron tos $(45,4 \%)$, fiebre $(39,7 \%)$ y expectoración $(35,8 \%)$ (Tabla 3$)$. La radiografía conocida en 144 casos mostraba alteraciones en $89,6 \%$, siendo las alteraciones radiológicas más frecuentes las opacidades $(72,9 \%)$ y los nódulos pulmonares $(8,5 \%)$. En 15 pacientes la radiografía fue normal $(11,6 \%)$.

Se encontró que los pacientes $\mathrm{VIH}+$ con $\mathrm{TBC}$ pulmonar tenían un promedio de CD4 menor y que todos tenían recuentos de CD4 $\leq 200$ células/mL y un menor uso de TAR. No se encontró diferencias en la proporción de candidiasis orofaríngea (Tabla 4).

En 111 FBC la indicación para su realización fue la sospecha de $\mathrm{TBC}$, la que se demostró en 12 casos (10,8\%); sin embargo, en 141 FBC solicitadas con otros diagnósticos diferentes de TBC se pesquisó esta enfermedad en 11 casos (7,8\%), lo que constituye el $47,8 \%$ de los casos de TBC pulmonar diagnosticados. La sensibilidad diagnóstica de la sospecha clínica de TBC pulmonar es de $52,2 \%$ y la especificidad de $56,8 \%$. De acuerdo a nuestros datos encontramos un bajo valor predictivo positivo (10,8\%) para el diagnóstico de TBC en base a la sospecha clínica previa a la FBC y un mejor valor predictivo negativo que alcanzó al 92,2\% (Tabla 5).

\section{Discusión}

La tuberculosis es una enfermedad muy relevante para las personas con infección por VIH por la frecuencia con que las afecta y por el permanente riesgo de reactivación de la infección tuberculosa latente. Este riesgo en infectados por 
Tabla 5. Valor predictivo de sospecha de tuberculosis pulmonar antes de la fibrobroncoscopía

\begin{tabular}{|lccc|}
\hline & Total casos & Casos con TBC pulmonar & Casos sin TBC pulmonar \\
Sospecha TBC pulmonar & 111 & 12 & 99 \\
Otros diagnósticos & 141 & 11 & 130 \\
Total & 252 & 23 & 229 \\
& & $\mathrm{VPP}^{*}=10,8 \%$ & $\mathrm{VPN}^{* *}=92,2 \%$ \\
\hline
\end{tabular}

*VPP: valor predictivo positivo. **VPN: Valor predictivo negativo.

el bacilo de la tuberculosis es de aproximadamente 5 a $10 \%$ anual, a diferencia de la población sin VIH que es de $10 \%$ durante toda la vida ${ }^{16}$. También se ha discutido el importante papel de la tuberculosis como causa de muerte en los infectados por VIH y la progresión del deterioro inmune que provoca la enfermedad. Por estas razones es necesario un diagnóstico oportuno de la TBC, especialmente si existe deterioro inmunológico, aunque debemos precisar que la tuberculosis puede producirse en infectados por VIH incluso sin deterioro inmune.

Desafortunadamente, entre el 40 y $60 \%$ de las personas con sospecha clínica o radiológica de tuberculosis no logra producir una muestra adecuada de esputo para el diagnóstico microbiológico, por lo que se puede recurrir a la broncoscopía para obtener muestras respiratorias, con rendimientos diagnósticos de $42,5 \%$ en países de alta incidencia de tuberculosis como India ${ }^{17}$ y Brasil $^{18}$ (44\%). Cuando las muestras proceden de lavado broncoalveolar la positividad es de $60 \%$ y esta sensibilidad aumenta a $84 \%$ al agregar biopsia transbronquial.

Nuestro estudio mostró que la tuberculosis es frecuente entre los infectados por VIH que fueron sometidos a broncoscopía (prevalencia de 9\%), lo que demuestra el aporte de este procedimiento, considerando que todos estos pacientes tenían previamente baciloscopías de esputo negativas. Nuestros resultados muestran un rendimiento menor a lo reportado por otros autores, que señalan como mínimo $26 \%$ de sensibilidad de la baciloscopía en lavado broncoalveolar ${ }^{19}$, lo que podría deberse a la menor incidencia de la tuberculosis en nuestro país y al hecho de no realizar lavado broncoalveolar en todos los casos, recogiendo en algunos de ellos sólo muestras de secreción broncoaspirada. Existen evidencias que demuestran un mayor rendimiento de las muestras de lavado broncoalveolar, las que según algunos reportes pueden alcanzar hasta un $90 \%$ de los diagnósticos de TBC pulmonar confirmados con los cultivos ${ }^{20}$. Además, en nuestros pacientes no realizamos biopsias transbronquiales y empleamos medios sólidos y no líquidos para los cultivos de micobacterias, en circunstancias que los medios líquidos tienen $22 \%$ más de rendimiento en muestras de esputo, llegando a detectar hasta el $84 \%$ de los casos demostrados de tuberculosis ${ }^{21}$. Por otra parte, nuestros procedimientos broncoscópicos se realizan con anestesia tópica con dimecaína, la que puede afectar el crecimiento del bacilo.

Destacamos la existencia de casos de tuberculosis que no fueron previamente sospechados en el 7,8\% de los pacientes sometidos a FBC por otros diagnósticos. Los enfermos que resultaron tener tuberculosis mostraron un menor nivel de recuento de linfocitos CD4 y con mayor frecuencia no recibían TAR. Es probable que este déficit de inmunidad no corregido tenga relación directa con la aparición de tuberculosis, por lo que recomendamos tener siempre presente la posibilidad de esta enfermedad en pacientes en esta situación de deterioro inmune. Se estima que existe un aumento de 2,1 veces en la probabilidad de tuberculosis en infectados por VIH por cada reducción de 200 células/mL en el recuento de $\mathrm{CD} 4{ }^{22}$. Como el $65,2 \%$ de los casos se demostraron a través de la baciloscopía, podría ser recomendable realizar esputo inducido en todos los pacientes con infección por VIH, independiente de la sospecha clínica de tuberculosis, bastando para ello sólo la presencia de síntomas respiratorios o alteraciones radiológicas.

La técnica del EI ha sido destacada en el diagnóstico de tuberculosis en personas que no producen esputo adecuado para baciloscopías. La realización de varios procedimientos de EI eleva la posibilidad diagnóstica de los cultivos desde $64 \%$ con una muestra a $91-99 \%$ con 3 muestras. Además, el EI contribuye a diagnosticar hasta el $30 \%$ de casos con radiología sugerente de tuberculosis activa y $31 \%$ en sintomáticos respiratorios, en ambos casos con baciloscopías y cultivos de Koch negativos en el esputo ${ }^{23}$. Efectuar EI previo a la broncoscopía reduce la necesidad de emplear este procedimiento invasivo y costoso. La técnica es relativamente sencilla y fácil de implementar a nivel ambulatorio. El Centro de 
Control de Enfermedades (CDC) de EE.UU. recomienda siempre realizar EI antes de la $\mathrm{FBC}^{24}$.

En nuestro estudio 34,8\% de los casos de tuberculosis se diagnosticaron mediante el cultivo de Koch a los 30 días de realizada la broncoscopía. A pesar de que se trata de casos con escasa carga bacilar, la demora en el diagnóstico puede ser relevante para pacientes inmunosuprimidos. Por esto es deseable incorporar otros métodos diagnósticos más precisos y rápidos, derivados de la biología molecular, al estudio de las muestras obtenidas del aparato respiratorio (reacción de polimerasa en cadena o PCR). Existen métodos automatizados de amplificación de regiones del ADN específicas del Mycobacterium tuberculosis (sondas genéticas) ${ }^{25}$ y últimamente se dispone en el medio nacional del geneXpert ${ }^{26}$ que a través de una PCR produce la amplificación de segmentos específicos de ADN de la micobacteria tuberculosa (IS6110) obteniendo resultados en 2 h. Además detecta cambios genéticos asociados a la resistencia a fármacos antituberculosos como rifampicina. Con el Xpert MTB/RIF se pueden detectar hasta el $80 \%$ de los casos de TBC pulmonar en infectados por VIH.

\section{Conclusiones}

La tuberculosis es más frecuente en los pacientes con infección por VIH. En nuestra experiencia en el $9 \%$ de los pacientes VIH+ sometidos a fibrobroncoscopía se demostró el diagnóstico de tuberculosis pulmonar. La sospecha de esta enfermedad en base a elementos clínicos tiene un reducido valor predictivo positivo. Se debe plantear el diagnóstico de tuberculosis ante la aparición de síntomas respiratorios y alteraciones radiológicas torácicas en inmunosuprimidos por VIH. Proponemos utilizar el esputo inducido para reducir el uso de fibrobroncoscopía, que es un procedimiento invasivo más costoso $\mathrm{y}$, en caso de realizar este procedimiento, disponer de las técnicas de la biología molecular para lograr un diagnóstico más precoz de la tuberculosis.

\section{Bibliografía}

1.- WORLD HEALTH ORGANIZATION. WHO Global Tuberculosis Report 2012.

2.- WORLD HEALTH ORGANIZATION. WHO Report 2009. Geneva: World Health Organization; 2009. Global Tuberculosis Control: surveillance, planning, financing.

3.- MCSHANE H. Co-infection with HIV and TB: double trouble. International Journal of STD \& AIDS 2005; 16 : 95-101.
4.- GETAHUN H, GUNNEBERG C, GRANICH R, NUNN P. HIV Infection-Associated Tuberculosis: the Epidemiology and the Response. Clin Infect Dis 2010; 50 (Suppl 3): S201-S207. doi: 10.1086/651492.

5.- MARTINSON N A, KARSTAEDT A, VENTER W D, OMAR T, KING P, MBENGO T, et al. Causes of death in hospitalized adults with a pre-mortem diagnosis of tuberculosis. An autopsy study. AIDS 2007; 21: 204350.

6.- WHALEN C, HORSBURGH C R, HOM D, LAHART C, SIMBERKOFF M, ELLNER J. Accelerated course of human immunodeficiency virus infection after tuberculosis. Am J Respir Crit Care Med 1995; 151: 129-35.

7.- PAWLOWSKI A, JANSSON M, SKÖLD M, ROTTENBERG M E, KÄLLENIUS G. Tuberculosis and HIV Co-Infection. PLoS Pathog 2012, Feb 8(2): e1002464. doi:10.1371/journal.ppat.1002464.

8.- HERRERA T. Evaluación nacional del programa de tuberculosis de Chile 2012. Informe MINSAL de Chile en evaluación del Comité Regional de Tuberculosis. SEREMI Metropolitano. 23 sept 2013.

9.- REVISED WHO clinical staging and immunological classification of HIV and case definition of HIV for surveillance. Ginebra. 2006.

10.- NORMA Y GUÍA TÉCNICA. Manual para el diagnóstico bacteriológico de la tuberculosis. Parte I Baciloscopía. Pág. 8 OPS 2008.

11.- NORMA Y GUÍA TÉCNICA. Manual para el diagnóstico bacteriológico de la tuberculosis. Parte II Cultivo. Pág. 9 OPS 2008.

12.- ANDERSON C, INHABER N, MENZIES D. Comparison of sputum induction with fiberoptic bronchoscopy in the diagnosis of tuberculosis. Am J Respir Crit Care Med 1995; 53: 1570-4.

13.- CONDE M B, SOARES S L, MELLO F C, REZENDE V M, ALMEIDA L L, REINGOLD A L, et al. Comparison of sputum induction with fiberoptic bronchoscopy in the diagnosis of tuberculosis. Am J Respir Crit Care Med 2000; 162: 2238-40.

14.- PEÑA C. Evaluación del programa de tuberculosis del Servicio de Salud Metropolitano Central de Chile 2012. Jornadas de Evaluación de Región Metropolitana y VI Región. Agosto 2013.

15.- HERRERA T. Evaluación nacional del Programa de Tuberculosis de Chile 2012. Informe MINSAL de Chile en Jornadas de Evaluación de Región Metropolitana y VI Región. Agosto 2013.

16.- CORBETT E L, DE COCK K M. Tuberculosis in the HIV-positive patient. Br J Hosp Med 1996; 56: 200-4.

17.- QUAISER S, AGARWAL A, KHAN R, HAQUE S F. Fiberoptic bronchoscopy, as a valuable diagnostic option in sputum negative pulmonary tuberculosis. A prospective study. Int J App Basic Med Res 2012; 2: 123-7.

18.- JACOMELLI M, SILVA P R, RODRÍGUEZ A J, DEMARZO S E, SEICENTO M, FIGUEIREDO V R. 
Bronchoscopy for the diagnosis of pulmonary tuberculosis in patients with negative sputum smear microscopy results. J Bras Pneumol 2012; 38; 2: 167-73.

19.- WORODRIA W, DAVIS J L, CATTAMANCHI A, ANDAMA A, DEN BOON S, YOO S D, et al. Bronchoscopy is useful for diagnosing smear-negative tuberculosis in HIV-infected patients. Eur Respir J 2010; 36: 446-56.

20.- BAUGHMAN R P, DOHN M N, LOUDON R G, FRAME P T. Bronchoscopy with bronchoalveolar lavage in tuberculosis and fungal infections. Chest 1991; 99: 92-7.

21.- MONKONGDEE P, MCCARTHY K D, CAIN K P, TASANEEYAPAN T, NGUYEN H D, NGUYEN T N, et al. Yield of acid-fast smear and mycobacterial culture for tuberculosis diagnosis in people with human immunodeficiency virus. Am J Respir Crit Care Med 2009; 180: 903-8.

22.- WILliams B G, DYE C. Antiretroviral drugs for tuberculosis control in the era of HIV/AIDS. Science. 2003; 301: 1535-7.
23.- MCWILLIAMS T, WELLS A U, HARRISON A C, LINDSTROM CAMERON R J, FOSKIN E. Induced sputum and bronchoscopy in the diagnosis of pulmonary tuberculosis. Thorax 2002, 57: 1010-4.

24.- JENSEN P. Guidelines for preventing the transmission of Mycobacterium tuberculosis in health-care settings, 2005. MMWRhttp://www.ncbi.nim.nih.gov/mmwr/ preview/mmwrhtml/rr5417a1.htm.

25.- LING D I, FLORES L L, RILEY L W, PAI M. Commercial nucleic-acid amplification tests for diagnosis of pulmonary tuberculosis in respiratory specimens: meta-analysis and meta-regression. PLoS One 2008, 3: e1536. doi:10.1371/journal.pone.0001536.

26.- WORLD HEALTH ORGANIZATION. WHO information note. February 2013. Xpert MTB/RIF increase timely TB detection among people living with HIV and saves live. www.who.int/tb/xpert_THIV_information_note.
Correspondencia a:

Dr. Carlos Peña M.

Hospital Clínico San Borja Arriarán

Santiago, Chile.

Email: carpemanti@hotmail.com 